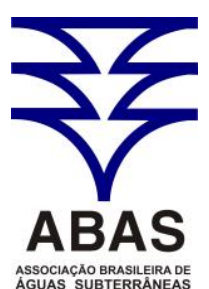

ASSOCIACÁO BRASILEIRADE
AGUAS SUBTERRANEAS www.abas.org

\title{
ERRO ASSOCIADO À SOLUÇÃO APROXIMADA DE DOMENICO EM CASOS REAIS DE CONTAMINAÇÃO E SUAS IMPLICAÇÕES PARA A QUANTIFICAÇÃO DE RISCO
}

\author{
ERROR OF APPROXIMATE DOMENICO SOLUTION IN ACTUAL \\ CONTAMINATION CASES AND ITS IMPLICATIONS FOR THE \\ RISK EVALUATION
}

\author{
Andreia Yoshinari', Elias Hideo Teramoto², Hung Kiang Chang ${ }^{3}$
}

Artigo recebido em: 19/09/2014 e aceito para publicação em: 03/11/2014.

DOI: http://dx.doi.org/10.14295/ras.v29i1.27966

\begin{abstract}
The approximate Domenico (1987) solution represents the analytical model of solute transport more widespread and accepted by environmental agencies, including quantifying risks to human health. Because it represents an approximation, a certain amount of error is to be expected. However, only from relatively recent works, these errors were investigated in a systematic manner, providing evidence that such errors can be highly significant depending on the parameters used. This study investigated the error of the Domenico (1987) solution in six actual cases of contamination by comparing with the exact solution of Wexler (1992). The results indicate that all parameters used in the Domenico (1987) model contribute in a greater or lesser extent to the presence of error. It is remarkable that the dimension of the source represents the factor that contribute most significantly to the error, followed by the longitudinal dispersion. The errors vary widely and in certain circumstances, as in regimes where hydrodynamic dispersion and advection have similar importance, can promote the underestimation of risk to human health, and consequently leading to bad decisions.
\end{abstract}

Keywords: Analytical solution. Risk analysis. Domenico solution. Wexler solution. Transport.

Resumo: A solução aproximada de Domenico (1987) representa o modelo analítico de transporte de soluto mais difundido e aceito por órgãos ambientais, inclusive para quantificação de riscos à saúde humana. Por representar uma aproximação, certa quantidade de erro deve ser esperada; contudo, somente a partir de trabalhos relativamente recentes, esses erros foram investigados de maneira sistemática, fornecendo indícios de que podem ser significativos a depender dos parâmetros empregados. Este trabalho investigou o erro da solução de Domenico (1987) em seis casos reais de contaminação a partir da comparação com a solução exata de Wexler (1992). Os resultados indicam que todos os parâmetros empregados no modelo de Domenico (1987) contribuem em maior ou menor proporção para a ocorrência de erro. Destaca-se que a dimensão da fonte representa o fator que contribui mais significativamente para o erro, seguida pela dispersão longitudinal. Os erros variam amplamente e, em certas circunstâncias como em regimes onde a dispersão hidrodinâmica e advecção possuem importâncias similares, podem promover a subestimação do risco à saúde humana, induzindo à tomada de decisões equivocadas.

Palavras-chave: Solução analítica. Análise de risco. Domenico. Wexler. Transporte.

\section{INTRODUÇÃO}

Métodos analíticos são amplamente adotados para estimar o potencial de transporte de contaminantes na água subterrânea e envolvem soluções de equações diferenciais parciais; aplicam-se a problemas de geometria simples e a aquíferos homogêneos. A despeito de suas limitações, as simulações empregando soluções analíticas de transporte podem ser empregadas de maneira preliminar e exploratória para uma inferência rápida do comportamento de contaminantes em águas subterrâneas.

Em problemas envolvendo o transporte de solutos no meio poroso, a avalição do regime de fluxo reinante é realizada mediante a quantificação do número de Peclet $(P e)$. Este parâmetro adimensional é relevante no estudo de fenômenos de transporte e é definida como sendo a razão da taxa de advecção em relação à taxa de

1 Programa de Pós-graduação em Geociências e Meio Ambiente, UNESP - Campus de Rio Claro (andreia.yoshinari@geoklock.com.br).

2 LEBAC - Laboratório de Estudo de Bacias, Departamento de Geologia Aplicada, UNESP - Campus de Rio Claro (eh_teramoto@yahoo.com.br).

${ }^{3}$ LEBAC - Laboratório de Estudo de Bacias, Departamento de Geologia Aplicada, UNESP - Campus de Rio Claro (chang@rc.unesp.br). 
dispersão hidrodinâmica e é assim definida (Bear, 1972)

$$
P e=v L / D
$$

Onde ${ }^{v}$ é a velocidade linear média (L/T); $L$ é a dimensão do fluxo (L) e D é a dispersão hidrodinâmica $\left(\mathrm{L}^{2} / \mathrm{T}\right)$.

Baixos valores de Pe indicam uma condição onde a dispersão hidrodinâmica (sobretudo a difusão) prevalece sobre a advecção. Em oposição, elevados valores de Pe indicam transporte com amplo predomínio da advecção.

Dentre as soluções analíticas de transporte disponíveis, a solução de Domenico (1987) representa o modelo mais difundido na atualidade. Esta

solução descreve um problema que admite uma

$$
\begin{aligned}
& C(x, y, z, t)=\frac{C_{0}}{8} \exp \left[\frac{v x}{2 D_{x}}\left(1-\sqrt{1+\frac{4 \lambda R D_{x}}{v^{2}}}\right)\right] \cdot \operatorname{erfc}\left[\frac{x-\frac{v t}{R} \sqrt{1+\frac{4 \lambda R D_{x}}{v^{2}}}}{2 \sqrt{D_{x} t / R}}\right] \\
& \left(\operatorname{erf}\left[\frac{y+W / 2}{2 \sqrt{\frac{D_{y x}}{v}}}\right]-\operatorname{erf}\left[\frac{y-W / 2}{2 \sqrt{\frac{D_{y} x}{v}}}\right]\right) \cdot\left(\operatorname{erf}\left[\frac{z+H / 2}{2 \sqrt{\frac{D_{z} x}{v}}}\right]-\operatorname{erf}\left[\frac{z-H / 2}{2 \sqrt{\frac{D_{z} x}{v}}}\right]\right)
\end{aligned}
$$

Onde: $C(x, y, z, t)$ é a concentração na posição $\mathrm{x}$, y e z e tempo t específico $\left(\mathrm{M} / \mathrm{L}^{3}\right)$; Co é a concentração no contorno de concentração especificada $\left(\mathrm{M} / \mathrm{L}^{3}\right) ; \mathrm{z}, \mathrm{y}, \mathrm{z}$ referem-se à posição nos eixos cartesianos $\mathrm{x}, \mathrm{y}$ e $\mathrm{z}$, respectivamente (L); t é o tempo (T); vx é a velocidade linear média de fluxo (L/T); ${ }^{\lambda}$ é o Coeficiente de decaimento de primeira ordem $\left(\mathrm{T}^{-1}\right) ; \mathrm{R}$ é o fonte como um plano vertical perpendicular ao fluxo horizontal, com dispersão mecânica longitudinal, transversal e vertical e taxa de decaimento de primeira ordem (Figura 1).

Segundo Guyonnet e Neville (2004), existem duas maneiras de estender a solução de uma fonte pontual para o caso de uma fonte planar e contínua de largura $L$ e altura $H$. A primeira abordagem é por meio de integração analítica, cuja integral deve ser avaliada numericamente, como por exemplo, a solução de Wexler (1992). A segunda, proposta por Domenico e Robbins (1985) e Domenico (1987) descreve uma aproximação, que combina duas soluções mais simplistas, uma para a dispersão no eixo $x$ e outra para a dispersão nos eixos $y$ e $z$. A solução proposta por Domenico (1987) é: retardamento; Dx, Dy, Dz são, respectivamente, os coeficientes de dispersão nas direções x,y e z; W é a largura do plano que representa o contorno de concentração especificada (L); H é a altura do plano que representa o contorno de concentração especificada (L); erf é a função de erro; $\operatorname{erfc}(x)$ é a função erro complementar.

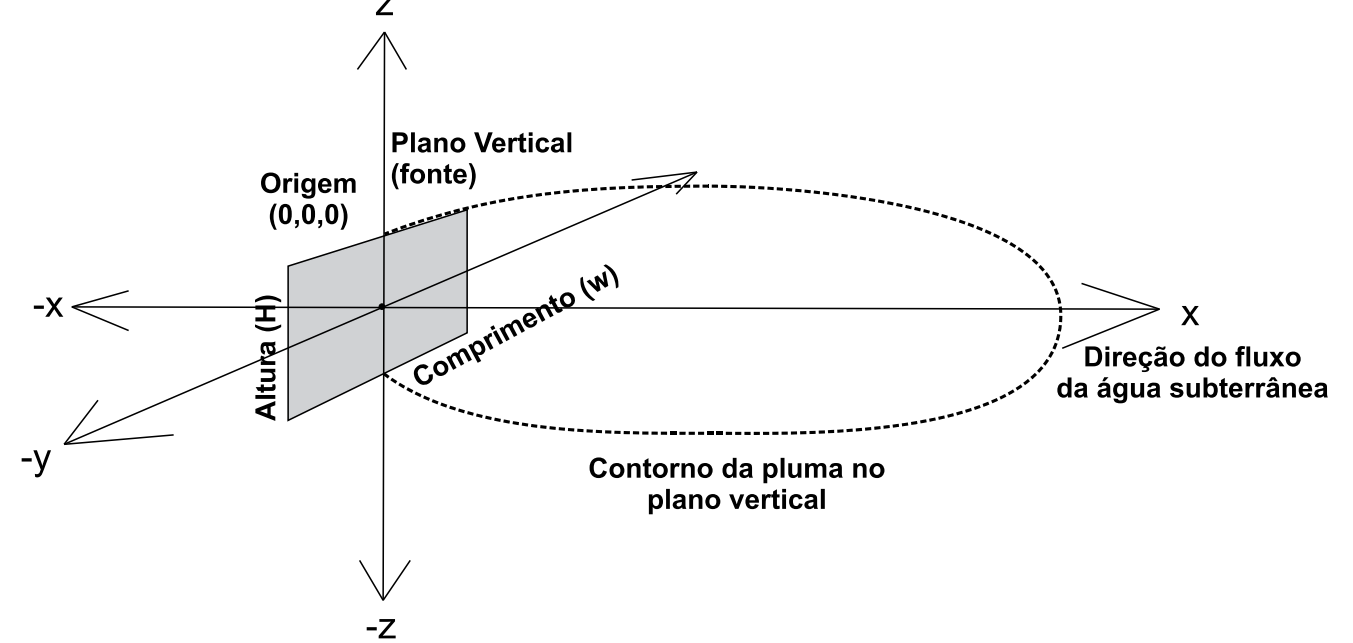

Figura 1 - Geometria e eixos do sistema para um plano vertical (fonte) que compõe as premissas da solução de Domenico (1987) (GUYONNET e NEVILLE, 2004)

Figure 1 - Geometry and axes of the system to a vertical plane at the source that makes up the premises of the solution of Domenico (1987) (GUYONNET AND NEVILLE, 2004) 
Em virtude de sua simplicidade e facilidade de implementação computacional, a solução proposta por Domenico (1987) se popularizou e se difundiu. Contudo, como é uma solução aproximada e não exata, uma quantidade finita de erro deve estar presente nesta solução.

Em oposição à solução aproximada de Domenico (1987) existem soluções exatas que descrevem o mesmo problema assumido nesta solução, como os modelos propostos por Sagar (1982) e Wexler (1992). A solução exata de Wexler (1992) para o problema de uma fonte planar em um domínio infinito pode ser obtida pela integração da equação ao longo da duração da liberação e sobre as dimensões da fonte nas direções $Y$ e $Z$ :

$$
C(x, y, z, t)=\frac{v C_{0} \exp \left[\frac{v x}{2 D_{x}}\right]}{8 \sqrt{\pi D_{x} / R}} \int_{0}^{t}\left\{\begin{array}{c}
\frac{1}{\tau^{1 / 2}} \exp \left[-\left(\lambda+\frac{v^{2}}{4 R D_{x}}\right) \tau-\frac{R x^{2}}{4 D_{x} \tau}\right] \\
\left(\operatorname{erfc}\left[\frac{y-W / 2}{2 \sqrt{D_{y} \tau / R}}\right]-\operatorname{erfc}\left[\frac{y+W / 2}{2 \sqrt{D_{y} \tau / R}}\right]\right) \cdot \\
\left(\operatorname{erfc}\left[\frac{z-H / 2}{2 \sqrt{D_{z} \tau / R}}\right]-\operatorname{erfc}\left[\frac{z+H / 2}{2 \sqrt{D_{z} \tau / R}}\right]\right)
\end{array}\right\} d \tau
$$

Onde: T é uma variável de integração em função do tempo.

A mensuração dos erros fornecidos pela solução de Domenico (1987) foi investigada sistematicamente somente em trabalhos relativamente recentes, a partir da comparação de seus resultados com soluções exatas. Dentro deste cenário, é possível destacar os trabalhos de Guyonnet e Neville (2004), Srinivasan et al. (2007) e West et al. (2007), cujos resultados permitiram compreender os fatores que fornecem erros à solução de Domenico (1987).

Srinivasan et al. (2007), investigando os erros associados à solução de Domenico (1987), verificou que nos casos em que a dispersividade longitudinal é igual a zero, esta solução pode ser considerada verdadeira. Entretanto, para valores de dispersividade longitudinal diferentes de zero, o erro pode alcançar valores muito significativos, como ilustra a Figura 2. Para esses autores, a depender da escolha dos parâmetros de entrada, o intervalo de erro varia de muito significativo (podendo alcançar 80\%) a negligenciável. Ainda segundo Srinivasan et al. (2007), as concentrações calculadas pela solução aproximada de Domenico (2007) são subestimadas quando comparadas à solução exata de Cleary e Ungs (1978) e Wexler (1992), o que a torna menos conservadora.

De acordo com CSMoS (2010), a dispersividade longitudinal é um parâmetro de calibração e não um parâmetro medido in situ. Portanto, os modelos baseados nas soluções de Domenico são razoáveis quando utilizados como ferramenta preliminar. No entanto, a seleção de uma dispersividade longitudinal baixa minimizaria o potencial erro.
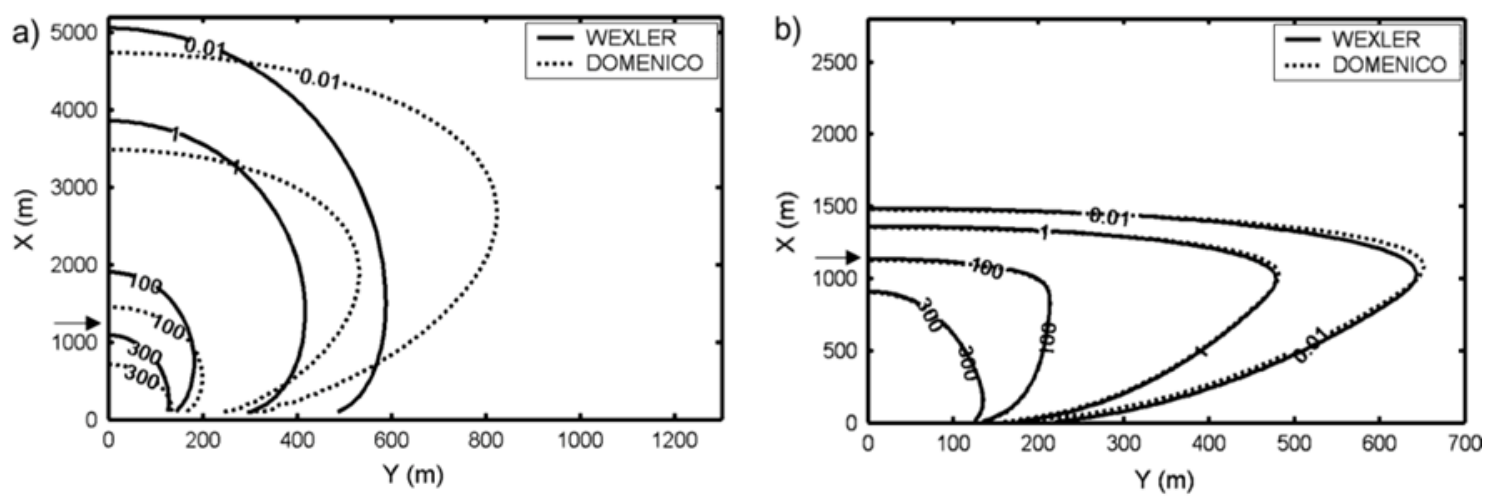

Figura 2 - Sensibilidade dos resultados para a variação dos valores de dispersividade longitudinal: soluções incluem isoconcentrações na frente advectiva para (a) ax=10 e (b) ax / 10 (SRINIVASAN et al., 2007)

Figure 2 - Sensitivity of results to variation of longitudinal dispersivity: isoconcentrações solutions include the advective front for (a) $\mathrm{x}=10$ and (b) $\mathrm{x} / 10$ (SRINIVASAN et al., 2007) 
Guyonnet e Neville (2004) apresentam comparações entre o modelo de Domenico (1987) e o de Sagar (1982), demonstrando que as discrepâncias entre os modelos são relativamente negligenciáveis ao longo da linha central da pluma de contaminação para regimes de fluxo dominados pela advecção e dispersão mecânica e não pela difusão molecular. Porém, aumentam significativamente fora do eixo central da pluma, principalmente quando se considera o decaimento e retardamento do contaminante. Verificaram, também, que a solução de Domenico (1987) mostra um erro pequeno quando o número de Peclet $(\mathrm{Pe})$ é maior do que 6, o que representa um aquífero relativamente permeável. Portanto, números altos de $\mathrm{Pe}$ significam transporte dominado pela advecção, enquanto valores menores de Pe podem indicar a influência da dispersão mecânica e de difusão molecular no transporte. CSMoS (2010) recomenda a utilização segura do modelo Domenico (1987) nas condições de transporte dominadas pela advecção (isto é, quando $\mathrm{Pe} \geq 10$ ), e cautela quando os processos de transporte são altamente influenciados pela dispersão (isto é, quando o aquífero é relativamente impermeável).

Por sua vez, West et al. (2007) assumiram uma abordagem mais conservadora e sugeriram que no contexto em que sejam determinadas as concentrações máximas admissíveis no local contaminado, o modelo de Domenico e Robbins (1985) pode subestimar as concentrações de maneira significativa ao longo do eixo central da pluma quando comparado a outros modelos com soluções exatas, tanto para condições transientes como steady-state, em domínios 2D ou 3D. Dessa forma, recomendam que modelos com solução exata devam ser preteridos ao modelo de Domenico (1987).

Srinivasan et al. (2007) e West et al.(2007) apresentaram um método matemático para derivar a solução de Domenico e explorar seus limites, o qual inclui a reinterpretação da variável tempo $(t)$ no termo da dispersão transversal, a partir da sua substituição por $x / v$.

A despeito de suas comprovadas limitações, a solução de Domenico é amplamente utilizada e aceita pelos órgãos ambientais, principalmente no Brasil e EUA. É o modelo de transporte adotado na planilha de Avaliação de Risco desenvolvida pela CETESB e publicada em seu site em 2009 e atualizada em 2013. Também é utilizado no software comercial Tool Kit Chemical Releases, desenvolvido pela Groundwater Services Inc., que constitui uma das ferramentas mais utilizadas por profissionais especializados em Análise de Risco à Saúde Humana no Brasil, sob a metodologia RBCA (Risk-Based Corrective Action).

Esse modelo de transporte também é utilizado nos pacotes de softwares BIOSCREEN, BIOCHLOR, FOOTPRINT e REMChlor distribuídos pelo Centro de Suporte de Modelagem em Subsuperfície (CSMoS - Center for Subsurface Modeling Suport) da USEPA (US Environmental Protection Agency). BIOSCREEN e REMChlor utilizam o modelo original de Domenico (1987) e BIOCHLOR e FOOTPRINT a versão modificada do modelo Martin-Hayden e Robbins (1997). Como alternativa para os aplicativos que empregam a solução de modelo, na atualidade existem aplicativos com soluções exatas, destacando-se o popular e difundido Bioscreen-AT descrito por Karanovic et al (2007), que emprega a solução de Sagar (1982)/Wexler (1992).

Face à comprovada existência de erro associado à solução de Domenico (1987), a proposta principal deste trabalho foi investigar o comportamento desse erro em condições hidrogeológicas distintas, com vistas a averiguar em quais contextos são mais significativos e quais suas implicações no gerenciamento de risco.

\section{METODOLOGIA EMPREGADA}

O erro gerado pela solução aproximada de Domenico (1987) é medido mediante a comparação com uma solução exata que descreva precisamente o mesmo problema, tendo sido adotada a solução de Wexler (1992) para tal comparação.

Embora trabalhos anteriores tenham conduzido análises para quantificação do erro na solução de Domenico (1987), tais trabalhos se restringiram à influência nos valores de velocidade de fluxo e dispersão mecânica. Pretendendo uma análise mais extensiva e abrangente, no presente trabalho foram contemplados todos os parâmetros exigidos por ambas as soluções. Para identificar em quais circunstâncias são verificados os maiores desvios da solução de Domenico (1987) em relação ao modelo de Wexler (1992), a análise do erro foi conduzida em diferentes cenários hidrogeológicos que congregam um conjunto de parâmetros de transporte distintos.

Para realizar esta comparação foi empregada uma versão modificada do aplicativo ETS3D (Exact Transport Solution 3D), desenvolvido pelo Laboratório de Estudo de Bacias (LEBAC/UNESP). A versão original do 
ETS3D trabalha exclusivamente com a solução de Wexler (1992). Contudo, para empreender a comparação aqui proposta foi elaborada uma versão que em prega adicionalmente a solução de Domenico (1987). O ETS3D modificado utiliza os parâmetros de entrada fornecidos e, a partir dos modelos de Wexler (1987) e Domenico (1987), calcula a concentração em uma malha de pontos definida para o modelo. A escolha do ETS3D se deveu ao fato dos resultados das simulações poderem ser exportados para diferentes arquivos de saída, tais como *.txt (arquivos de texto) e *.grds (grid do Surfer), que permite flexibilidade e facilidade operacional para quantificação da discrepância das duas soluções aqui comparadas.

A estimativa do erro é realizada por meio da comparação dos resultados de ambas as soluções analíticas empregadas para os mesmos parâmetros de entrada. Após as simulações, em cada ponto da malha de pontos do modelo o erro é quantificado partir da relação expressa abaixo:

$$
\text { Erro relativo }(\%)=\left[\frac{C_{\text {Domenico }}-C_{W \text { exler }}}{C_{\text {Wexler }}}\right] \times 100
$$

Onde: $C_{\text {Domenico }}$ é a Concentração calculada empregando a solução de Domenico (1987) (mg/l); $C_{\text {Wexler }}$ é a Concentração calculada empregando a solução exata de Wexler (1992) (mg/l).

Os valores de erro relativo médio em todo o domínio simulado foram calculados pela média aritmética de todos os valores de concentração.

A análise de sensibilidade relativa $\left(S_{\mathrm{r}}\right)$ foi realizada para o erro relativo médio entre os dois modelos analíticos, considerando-se a variação do valor de cada parâmetro de entrada. Para tal análise, foi empreendida uma sucessiva alteração de $+/-10 \%$ em cada parâmetro individualmente, enquanto os demais permaneciam constantes. Todos os parâmetros requeridos por ambos os modelos foram testados. A sensibilidade relativa foi determinada de acordo com a metodologia de McCuen e Snyder (1986), pela equação:

$S_{r}=\left[\begin{array}{l}\frac{\mathscr{S}}{S_{\tilde{i}}} \\ \frac{\mathbb{M E}}{E_{\mathrm{i}}}\end{array}\right]$

Onde: $S_{\mathrm{r}}$ é a sensibilidade relativa do modelo aos parâmetros de entrada; $\Delta \mathrm{S}$ é a taxa de variação da resposta do modelo; $S_{i}$ é o valor inicial de saída; $\Delta \mathrm{E}$ é a taxa de variação da entrada dos dados; $E_{i}$ é o valor inicial do parâmetro de entrada.

$\mathrm{Na}$ equação acima, o valor de $\mathrm{S}_{\mathrm{r}}$ é definido como a razão entre a taxa de variação da resposta do modelo $\left({ }^{\Delta} \mathrm{S}\right)$ a uma pequena variação na entrada $\left({ }^{\Delta} \mathrm{E}\right)$, normalizadas pelos valores iniciais da saída $\left(\mathrm{S}_{\mathrm{i}}\right)$ e da entrada $\left(\mathrm{E}_{\mathrm{i}}\right)$, respectivamente.
De acordo com Chaves (2009), os valores de sensibilidade relativa podem ser interpretados da seguinte forma: se $|\mathrm{Sr}|>1,5$ o modelo apresenta alta sensibilidade ao parâmetro; se $|\mathrm{Sr}|<0,5$ a sensibilidade é baixa; se $0,5<|\mathrm{Sr}|<1,5$ a sensibilidade é intermediária. Valores próximos a zero indicam que o modelo é relativamente insensível ao parâmetro.

\section{ESTUDO DE CASOS}

O estudo de casos aqui empreendido utilizou dados disponibilizados pela empresa Geoklock Consultoria e Engenharia Ambiental Ltda., sob termo de confidencialidade. Inicialmente foi realizada uma avaliação do quadro ambiental de diversos casos, cujos contaminantes são compostos orgânicos voláteis, preferencialmente do grupo dos aromáticos e etenos clorados.

A primeira triagem enfocou a análise das características geológicas, hidrogeológicas e da contaminação para a definição de quais áreas são mais apropriadas para os objetivos deste estudo. Também foram verificados os dados referentes aos parâmetros de entrada utilizados na simulação do transporte de soluto, tais como: i) dimensão da fonte de contaminação; ii) concentração na água subterrânea; iii) dispersividade (alfa x, y e z); iv) condutividade hidráulica; v) gradiente hidráulico; vi) fator de retardamento; vii) porosidade efetiva; e viii) taxa de decaimento.

As avaliações efetuadas resultaram na escolha de seis áreas, que atenderam aos requisitos necessários para execução dos testes pretendidos, no que concerne à disponibilidade de informações. A Tabela 1 apresenta as características hidrogeológicas e contaminantes presentes nos seis casos analisados no presente trabalho. 
Tabela 1 - Características gerais de cada caso estudado, e parâmetros de entrada empregados nas simulações utilizando os modelos de Wexler (1992) e Domenico (1987)

Table 1 - General characteristics of each case study and input parameters used in the simulations using the model Wexler (1992) and Domenico (1987)

\begin{tabular}{|c|c|c|c|c|c|c|}
\hline Parâmetros & Caso 1 & Caso 2 & Caso 3 & Caso 4 & Caso 5 & Caso 6 \\
\hline Litologia do Aquífero & $\begin{array}{c}\text { Areia média a } \\
\text { grossa }\end{array}$ & Silte argiloso & $\begin{array}{c}\text { Aterro silte } \\
\text { argiloso }\end{array}$ & Silte argiloso & Areia argilosa & Argila arenosa \\
\hline Composto de interesse & Benzeno & Benzeno & Benzeno & Xilenos & Benzeno & Benzeno \\
\hline Concentração (mg/l) & 16,4 & 0,566 & 0,453 & 13,33 & 0,153 & 9,45 \\
\hline Dimensão da Fonte em $Y$ & 20 & 3 & 8 & 10 & 5 & 10 \\
\hline Dimensão da Fonte em Z & 3 & 2 & 3 & 2 & 3 & 3 \\
\hline $\operatorname{ax}(\mathrm{m})$ & 1 & 10 & 1 & 10 & 1,5 & 5 \\
\hline ay $(\mathrm{m})$ & 0,01 & 0,1 & 0,01 & 0,1 & 0,075 & 0,04 \\
\hline$a z(m)$ & 0,001 & 0,01 & 0,0001 & 0,01 & 0,0075 & 0,004 \\
\hline Gradiente Hidráulico & 0,007 & 0,075 & 0,014 & 0,048 & 0,014 & 0,01 \\
\hline Porosidade efetiva & 0,3 & 0,15 & 0,2 & 0,15 & 0,1 & 0,17 \\
\hline $\begin{array}{l}\text { Decaimento de primeira ordem } \\
\qquad\left(\text { dias }^{-1}\right)\end{array}$ & 0,08 & 0,0038 & 0,003 & 0,0038 & 0,0008 & 0,0001 \\
\hline
\end{tabular}

\section{RESULTADOS}

As simulações utilizando o aplicativo ETS3D modificado e os parâmetros compilados na Tabela 1, para cada um dos seis casos, permitiram comparar os desvios entre as soluções de
Domenico (1987) e Wexler (1992) a partir da Equação 3. Os mapas de distribuição dos erros relativos calculados encontram-se ilustrados nas Figuras 3 a 8 .

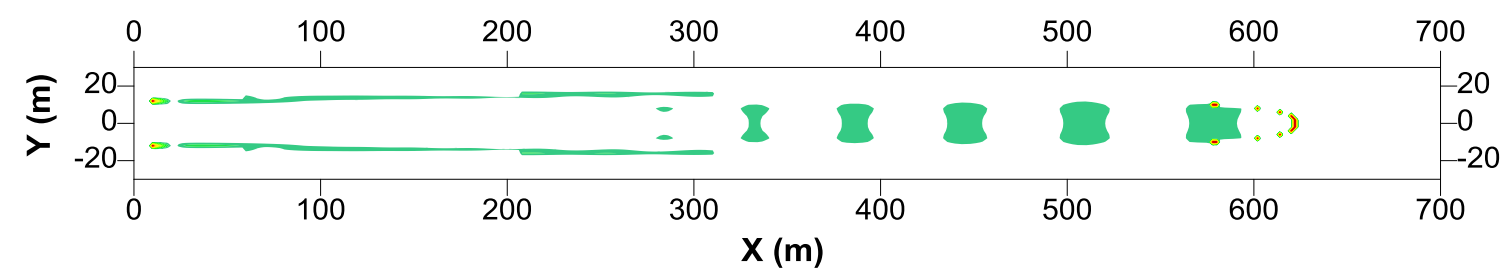

Erro relativo (\%)

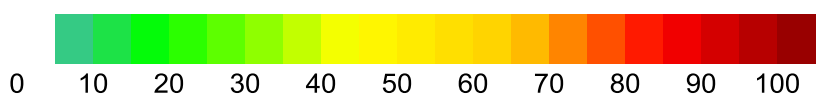

Figura 3 - Mapa com a distribuição do erro relativo para o Caso 1

Figure 3 - Map showing the distribution of the relative error for Case 1 
YOSHINARI, A.; TERAMOTO, E. H.; CHANG, H.K.

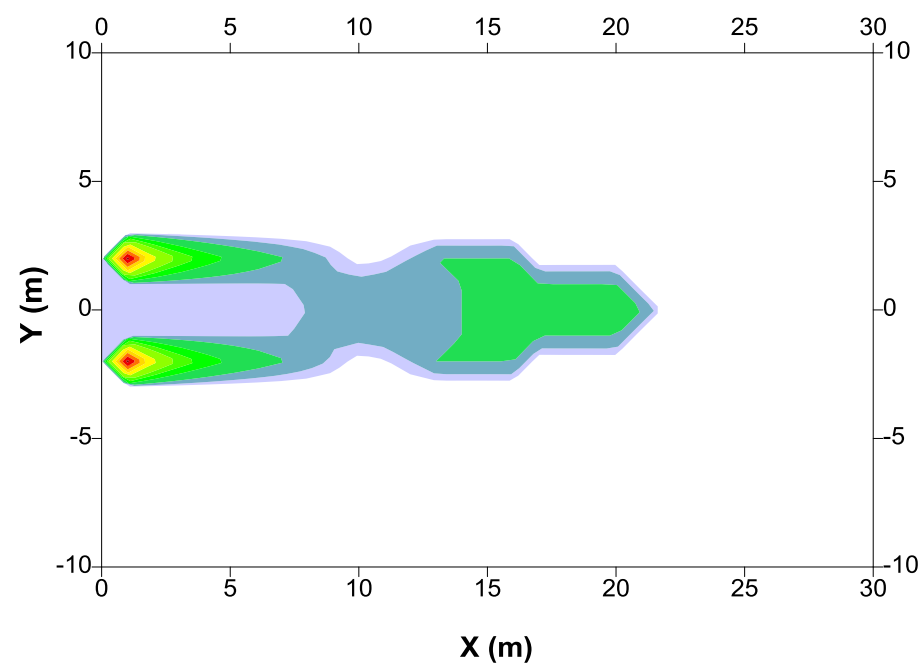

Erro relativo (\%)

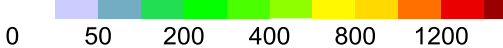

Figura 4 - Mapa com a distribuição do erro relativo para o Caso 2

Figure 4 - Map showing the distribution of the relative error for Case 2

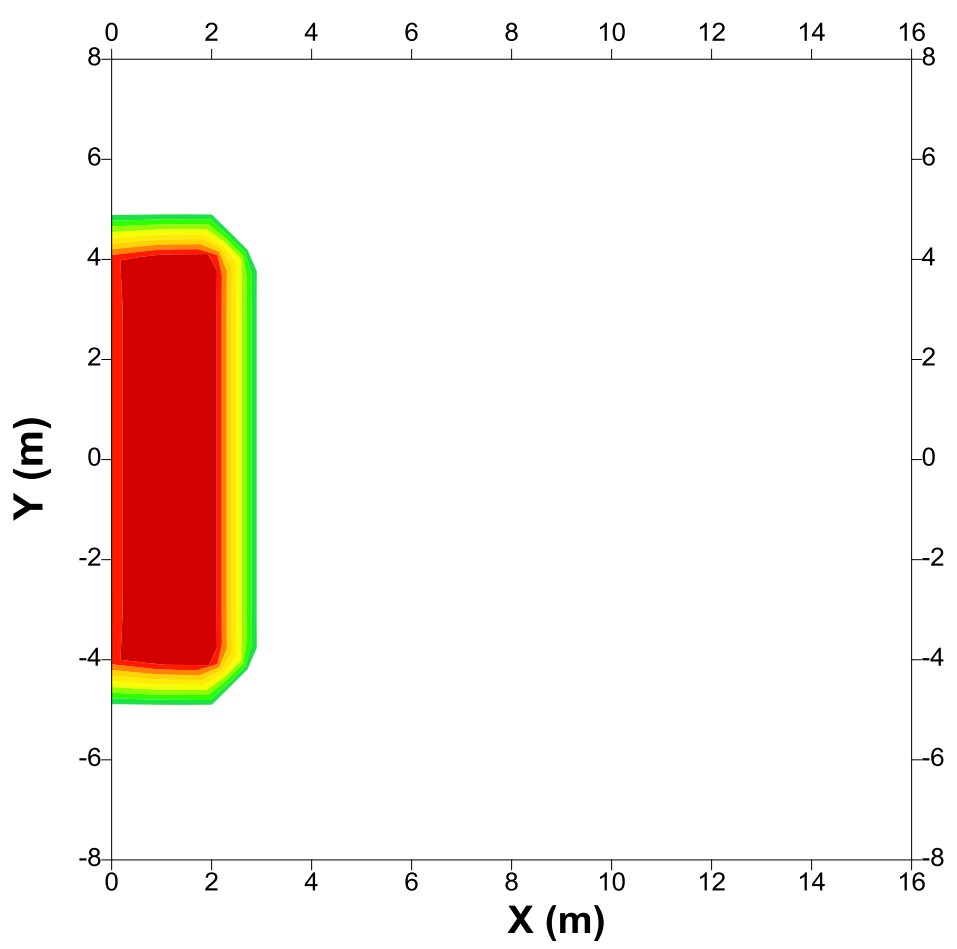

\section{Erro relativo (\%)}

$\begin{array}{lllllllllll}0 & 10 & 20 & 30 & 40 & 50 & 60 & 70 & 80 & 90 & 100\end{array}$

Figura 5 - Mapa com a distribuição do erro relativo para o Caso 3

Figure 5 - Map showing the distribution of the relative error for Case 3 

quantificação de risco

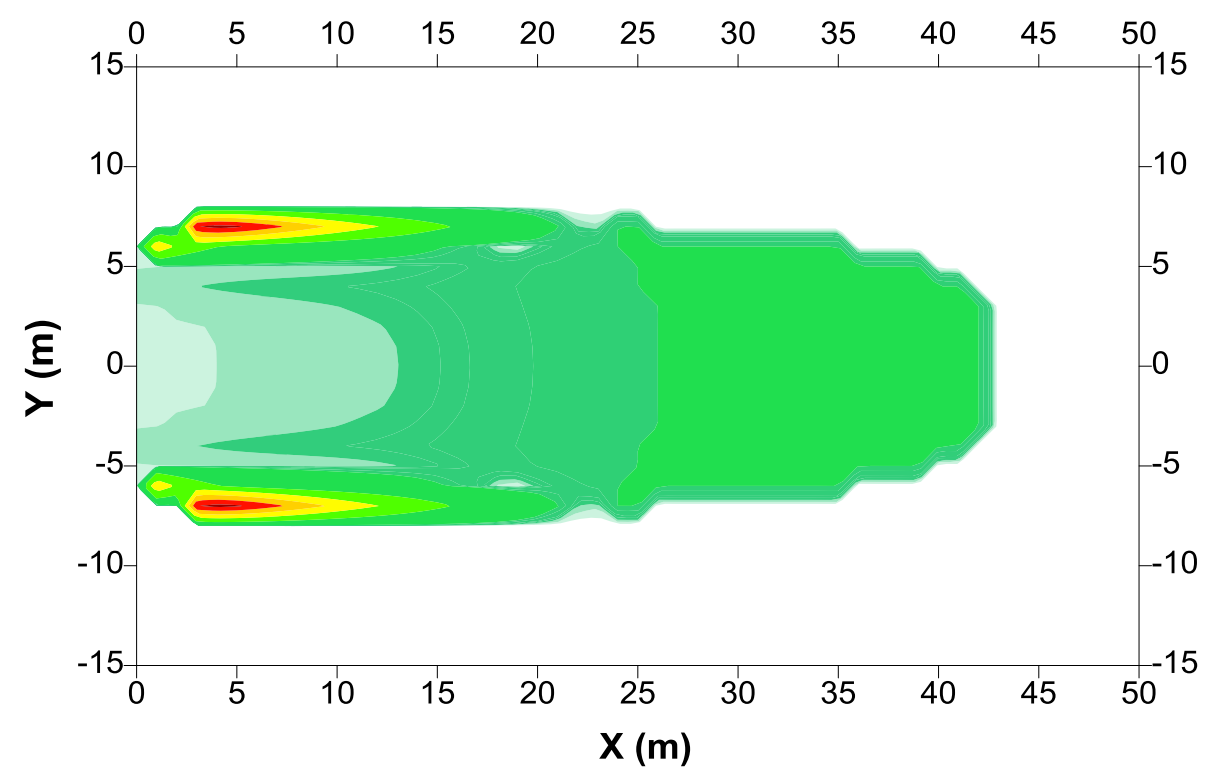

\section{Erro relativo (\%)}

$\begin{array}{lllllll}0 & 20 & 40 & 75 & 1000 & 3000 & 5000\end{array}$

Figura 6 - Mapa com a distribuição do erro relativo para o Caso 4 Figure 6 - Map showing the distribution of the relative error for Case 4

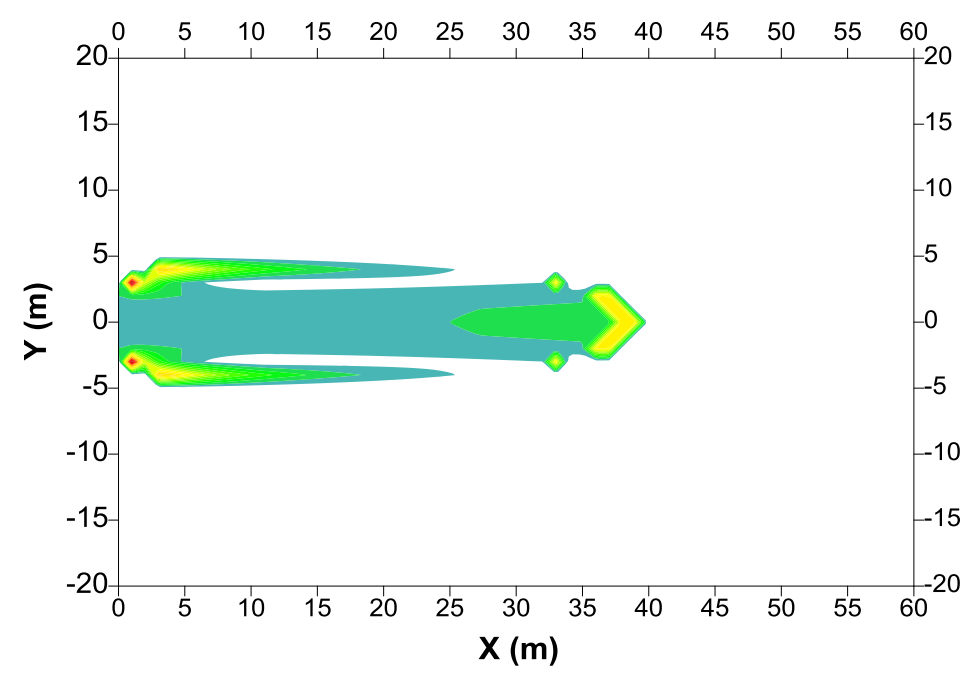

Erro relativo (\%)

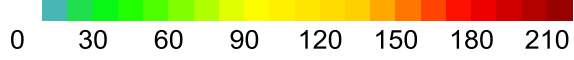

Figura 7 - Mapa com a distribuição do erro relativo para o Caso 5

Figure 7 - Map showing the distribution of the relative error for Case 5 


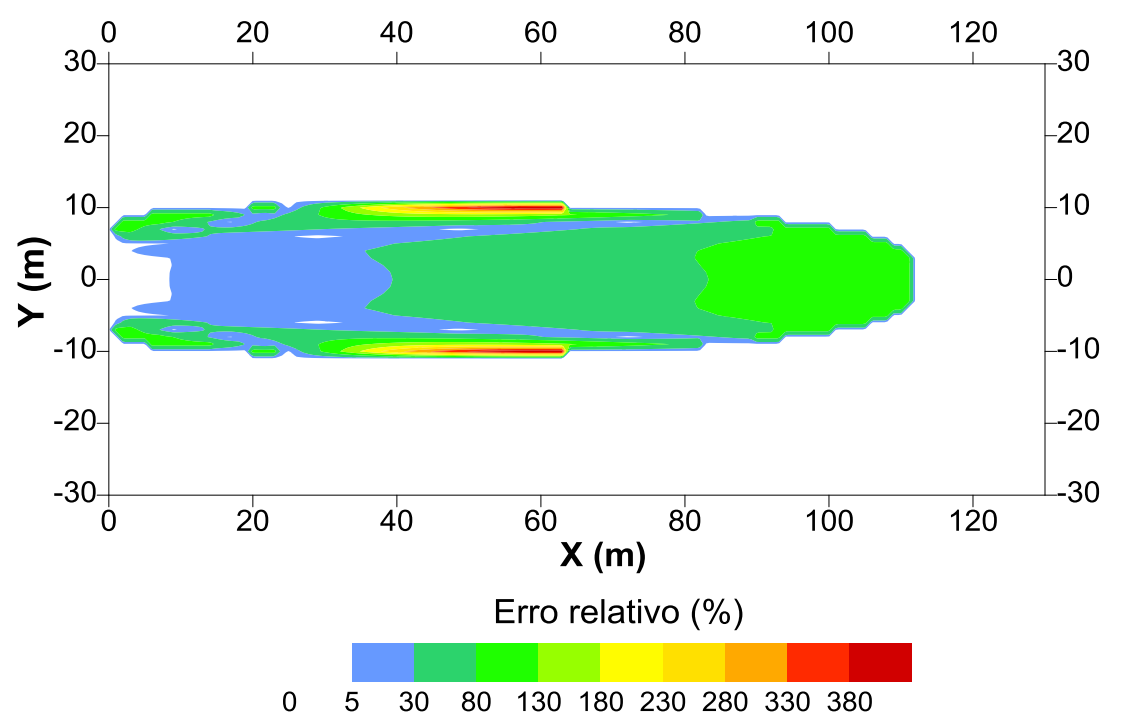

Figura 8 - Mapa com a distribuição do erro relativo para o Caso 6

Figure 8 - Map showing the distribution of the relative error for Case 6

Com os valores de erro da solução de Domenico (1987) estimados, foi calculado o erro relativo médio, que consistiu na média aritmética do erro para todos os pontos onde a concentração é superior a 0 na solução de Wexler (1992). Os valores de erro médio calculados para os casos estudados apresentam ampla variação (Tabela 2).

Tabela 2 - Valores de Erro Relativo Médio para cada caso estudado

Table 2 - Values of Average Relative Error for each study case

\begin{tabular}{ll}
\hline Caso & Erro Relativo Médio (\%) \\
\hline 1 & 1,64 \\
2 & 18,62 \\
3 & 8,90 \\
4 & 93,90 \\
5 & 1,23 \\
6 & 15,93 \\
\hline
\end{tabular}

Para investigar a origem da fonte desses apresentados pela dispersividade longitudinal, erros foi calculado o coeficiente de correlação entre o Erro e os parâmetros de entrada dos modelos empregados; os resultados são apresentados na Tabela 3. Os valores de coeficiente de correlação mais expressivos são transversal e vertical, sugerindo que na variação de tais parâmetros reside a maior fonte de desvios entre as duas soluções testadas, com destaque para a dispersividade longitudinal.

Tabela 3 - Valores de coeficiente de correlação do erro relativo para os parâmetros de entrada empregados nas simulações.

Table 3 - Values of correlation coefficient for the relative error in the input parameters used in the simulations.

\begin{tabular}{ll}
\hline Parâmetro & Coeficiente de correlação \\
\hline Kxx & 0,096 \\
Dx & 0,520 \\
Dy & 0,334 \\
Dz & 0,322 \\
1 & 0,077 \\
Dimensão da fonte em Y & 0,002 \\
Concentração & 0,152 \\
\hline
\end{tabular}




\section{Análise de sensibilidade}

Adotando os procedimentos descritos previamente, foram realizadas análises de sensibilidade com vistas a associar as variações do erro a parâmetros específicos empregados pela solução de Domenico (1987). Os resultados da análise de sensibilidade são apresentados na Tabela 4.

Tabela 4 - Resultado da análise de sensibilidade relativa $\left(S_{\mathrm{r}}\right)$

Table 4 - Results of the relative sensitivity analysis (Sr)

\begin{tabular}{|c|c|c|c|c|c|c|c|}
\hline \multicolumn{2}{|l|}{ Velocidade (m/ano) } & 225 & 1,8 & 0,3 & 3 & 30 & 4 \\
\hline $\begin{array}{l}\text { Parâmetro de } \\
\text { Entrada }\end{array}$ & $\begin{array}{c}\text { Variação do Parâmetro } \\
\text { de Entrada }\end{array}$ & \multicolumn{6}{|c|}{ Sensibilidade Relativa (Sr) } \\
\hline Alpha $x$ & $-10 \%$ & 0,6 & 0,9 & 0,0 & 1,0 & 0,4 & 0,8 \\
\hline \multirow{2}{*}{ Alpha y } & $10 \%$ & 0,2 & $-0,5$ & 0,0 & $-0,5$ & $-0,4$ & $-0,1$ \\
\hline & $-10 \%$ & 0,7 & $-0,7$ & 0,0 & $-0,5$ & 0,3 & 0,4 \\
\hline Alpha z & $10 \%$ & 0,1 & 0,0 & 0,0 & 0,0 & $-0,2$ & 0,0 \\
\hline Kxx & $-10 \%$ & 0,5 & $-0,2$ & $-0,1$ & $-1,2$ & 0,3 & 0,6 \\
\hline \multirow{2}{*}{ Decaimento } & $10 \%$ & $-0,3$ & 0,0 & 0,1 & 1,2 & $-0,3$ & 0,0 \\
\hline & $-10 \%$ & $-0,5$ & 0,0 & $-2,7$ & 0,6 & $-0,1$ & 0,0 \\
\hline \multirow{2}{*}{ Dimensão da fonte $Y$} & $10 \%$ & 0,7 & $-2,7$ & 0,0 & 0,1 & $-0,9$ & $-0,4$ \\
\hline & $-10 \%$ & 0,6 & $-4,4$ & 2,2 & 4,0 & $-0,5$ & 1,2 \\
\hline \multirow{2}{*}{ Dimensão da fonte $Z$} & $10 \%$ & $-0,3$ & 0,0 & 0,0 & 0,1 & $-0,1$ & 0,0 \\
\hline & $-10 \%$ & $-0,1$ & 0,0 & 0,0 & 0,1 & 0,2 & 0,0 \\
\hline
\end{tabular}

Legenda:

$|\mathrm{Sr}|>1,5$ : elevada

$0,5<|\mathrm{Sr}|<1,5$ : intermediária

$|\mathrm{Sr}|<0,5$ : baixa

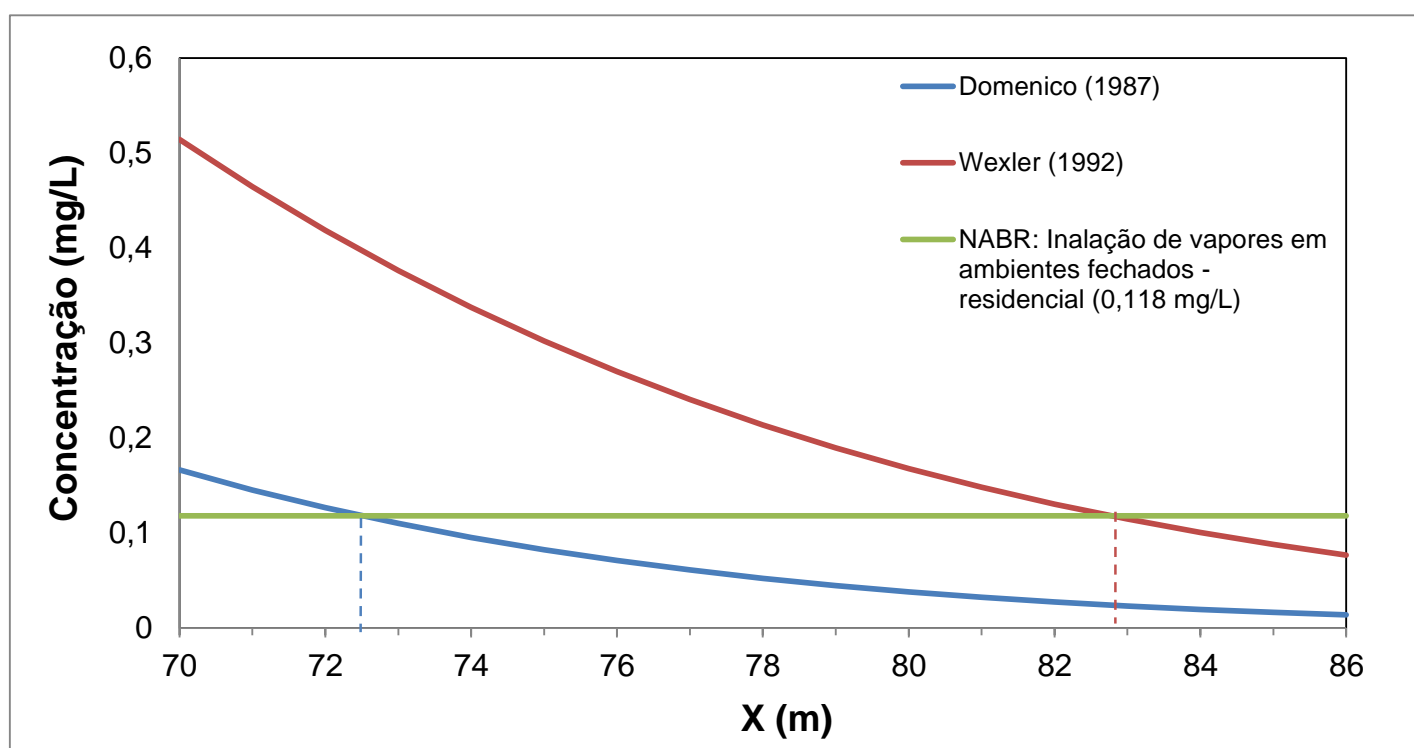

Figura 9 - Distribuição das concentrações de benzeno ao longo da linha central da pluma $(Y=0)$ frente ao NABR do composto no Caso 6 
Figure 9 - Distribution of concentrations of benzene along the plume centerline $(Y=0)$ of the compound across the $\mathrm{NaBr}$ in Case 6

\section{Análise da Tomada de Decisão}

Com o objetivo de avaliar se os resultados obtidos pelos dois modelos acarretariam em uma tomada de decisão diferente no gerenciamento do passivo ambiental, foram avaliadas as concentrações ao longo da linha central da pluma $(\mathrm{Y}=0)$ frente ao NABR (Nível Aceitável com Base no Risco, definido por CETESB, 2005) do composto de interesse, nos casos aqui considerados benzeno e xilenos.

Os resultados indicam que a diferença entre o modelo de Domenico (1987) e o de Wexler (1992) é de no máximo 2,0 m de distância, sendo que o Domenico (1987) é sempre menos conservador, ou seja, indica que a concentração do NABR é atingida em uma distância menor que a de Wexler (1992) nos Casos 1 a 5 .

A diferença entre os dois modelos, nos casos estudados, pode ser considerada pouco significativa, pois na prática o receptor estaria exposto ou muito próximo a um potencial risco de inalação de vapores orgânicos provenientes da água subterrânea, merecendo para ambos os modelos um plano de ação com vistas à proteção destes receptores. Dessa forma, a escolha de qualquer um dos modelos acarretaria em uma mesma decisão no gerenciamento do passivo ambiental.

Por outro lado, o Caso 6, ilustrado na Figura 9, acusa uma diferença de aproximadamente $10 \mathrm{~m}$ de distância entre os dois modelos comparados, uma vez que a maior diferença de concentração entre ambos está no meio da pluma, porção que corresponde ao valor do NABR adotado. Esse caso corresponde a um aquífero de permeabilidade intermediária, em que possivelmente

processos de dispersão hidrodinâmica e advecção têm igual importância e apresentam os resultados mais discrepantes entre a solução de Domenico (1987) e Wexler (1992).

\section{DISCUSSÃO DOS RESULTADOS}

Os testes aqui conduzidos indicam que os erros nas estimativas de concentração calculadas pela solução aproximada de Domenico (1987) podem ser bastante expressivos, dependendo das características da área estudada.

O erro presente na solução de Domenico (1987) é amplamente variável, como observado na Tabela 4, e não pode ser previsto a priori. A despeito desse fato, verifica-se que os maiores valores de erros estão associados à dispersividade [, sobretudo a dispersividade longitudinal (ax), em concordância com as observações realizadas por Guyonnet e Neville (2004), Srinivasan et al. (2007) e West et Al. (2007).

Os valores reunidos na Tabela 4 mostram que o erro é sensível a aos parâmetros do modelo, indicando que todos esses parâmetros contribuem em maior ou menor grau para a existência de discrepância entre a solução de Domenico (1987) e Wexler (1992). Surpreendentemente, o parâmetro que contribuiu para o incremento do erro foi o parâmetro "Dimensão da fonte em Y", que correspondente a largura do contorno de concentração constante. Assim, a análise de sensibilidade corroborou as observações anteriores de que a dispersividade longitudinal exerce forte influência no incremento do erro da solução de Domenico (1987).

Para regimes de baixa velocidade de fluxo, como a observada no estudo de Caso 3, o erro mostra-se sensível à condutividade hidráulica na direção $\mathrm{x}$ e ao coeficiente de decaimento de primeira ordem. Esse comportamento não havia sido discutido em trabalhos prévios envolvendo a quantificação do erro na solução de Domenico (2007), de forma que merece ser mais bem entendido.

No que concerne à quantificação do risco à saúde humana, adotando como referência o NABR definido pela CETESB (2006), verifica-se que a adoção do modelo de Domenico (1987) deve ser aplicada com cautela para aquíferos de permeabilidade baixa a intermediária, em que possivelmente os processos de advecção e de dispersão hidrodinâmica têm igual importância. A diferença entre os dois modelos pode ser considerada significativa nos casos de áreas contaminadas com características similares às observadas no Caso 6. Verifica-se que, dependendo da localização do receptor em relação à fonte, o resultado de Wexler (1992) pode sugerir a existência de risco à saúde humana, enquanto o modelo de Domenico (1987) pode sugerir a inexistência de risco.

\section{CONCLUSÕES}

Este trabalho buscou comparar os resultados fornecidos pela solução aproximada de Domenico (1987) com os da solução exata de 
Wexler (1992), aplicados a problemas reais envolvendo contaminação por compostos monoaromáticos. Os resultados encontrados coadunam-se com as observações encontradas nos trabalhos de West et al. (2007), Srinivasan et al. (2007) e Guyonet e Neville (2004), que afirmam que a acurácia da solução de Domenico (1987) é altamente dependente dos parâmetros de entrada empregados.

A quantificação do erro presente na solução de Domenico (1987) baseou-se na comparação com a solução exata de Wexler (1992), associada à análise de sensibilidade de cada parâmetro empregado pelo modelo, com o intuito de se atribuir pesos relativos a cada parâmetro para o incremento do erro na solução analítica testada.

Em determinadas circunstâncias em que a advecção e a dispersão hidrodinâmica longitudinal têm igual importância, a adoção de modelos que empregam a solução de Domenico (1987) pode subestimar o erro envolvido e induzir

\section{REFERÊNCIAS}

BEAR, J. Hydraulics of groundwater Nova Iorque: McGraw-Hill. 1972.

CHAVES, H. M. L. Sensibilidade do Modelo Hydrus aos Parâmetros Hidráulicos do Solo em Diferentes Texturas. Revista Brasileira de Recursos Hídricos - RBRH. Brasília, v. 14, n. 2, p. 33-37, abr./jun. 2009.

CENTER FOR SUBSURFACE MODELING SUPORT CSMoS. Potential Limitations of Four Domenico-Based Fate and Transport Models. 2010. Disponível em: http://www.epa.gov/ada/csmos/domenico.html. Acesso em 31 ago. 2014.

COMPANHIA DE TECNOLOGIA DE SANEAMENTO AMBIENTAL (CETESB). Ações Corretivas Baseadas em Risco (ACBR) Aplicadas a Áreas Contaminadas com Hidrocarbonetos Derivados de Petróleo e Outros Combustíveis Líquidos - Procedimentos. São Paulo:

CETESB, 2006.

DOMENICO, P.A. An Analytical Model for Multidimensional Transport of Decaying Contaminant Species. In: Journal of Hydrology, v. 91, p. 45-58, 1987.

GUYONNET, D.; NEVILLE, C. Dimensionless Analysis of Two Analytical Solutions for 3-D Solute Transport in à tomada de decisões equivocadas. Desse modo, por cautela, não se recomenda a utilização da solução de Domenico (1987) para quantificação do risco à saúde humana.

Ressalta-se que a solução aproximada de Domenico (1987) se popularizou em virtude desta solução não necessitar realizar cálculos numéricos para aproximar a integração de massa em função de tempo, exigida pelas soluções analíticas exatas. A aproximação numérica de uma integral era computacionalmente dispendiosa à época em que o modelo de Domenico (1987) foi proposta. Contudo, em virtude do poder de processamento dos computadores atuais, a execução de soluções analíticas exatas exige pouco tempo de processamento e a simplicidade do modelo de Domenico (1987) deixou de ser vantajosa. Desse modo, em função dos erros observados e da abundância de soluções exatas disponíveis, a adoção do modelo de Domenico (1987) não mais se justifica.

Ground Water. Journal of Contaminant Hydrology. v.75, p.142-153. 2004.

Karanovic, M.; Neville, C. J.; Andrews, C. B. BIOSCREENAT: BIOSCREEN with an Exact Analytical Solution. Groundwater, v. 45, n. 2, p. 242-245, 2007.

SAGAR, B. Dispersion in Three Dimensions: Approxi-mate Analytical Solutions. In: Journal of Hydraulics Division, American Society of Civil Engineers. v. 108, n.HY1, p.4762. 1982.

SRINIVASAN, V.; CLEMENT, T.P.; LEE, K.K. Domenico Solution - Is it Valid? In: GroundWater. Vol. 45, n.2, p. 136-146. 2007.

WEST, M.R.; KUEPER, B.H.; UNGS, M.J. On the Use and Error osAproximation in the Domenico (1987) Solu tion. In: Ground Water. Vol. 45, No 2, p.126-135. 2007.

Wexler, E. J. Analytical Solutions for One-, Two-, and Three-Dimensional Solute Transport in Ground Water Systems With Uniform Flow. In: Techniques of Water Resources Investigations of the United States Geological Survey. Chapter B-7 in Book 3, Applications of Hydraulics, p. 79. 1992 